\title{
Hyperhidrosis as the presenting symptom in post-traumatic syringomyelia*
}

\author{
F E Glasauer $\mathrm{MD}^{1 \dagger} \mathrm{J} \mathrm{J}$ Czyrny $\mathrm{MD}^{2}$ \\ ${ }^{1}$ Department of Neurosurgery, ${ }^{2}$ Department of Rehabilitation Medicine, State University \\ of New York at Buffalo School of Medicine and Biophysical Sciences, Buffalo, New \\ York, USA.
}

\begin{abstract}
Post-traumatic syringomyelia is now a well known entity and occurs months or years after a spinal cord injury. The presenting symptoms are usually pain, progressive motor weakness, sensory changes, and increased spasticity. Profuse sweating or hyperhidrosis can be a symptom of the post-traumatic syrinx or can occur in autonomic dysreflexia provoked by peripheral stimuli. We present two patients with cervical spine fractures whose presenting symptom of posttraumatic syringomyelia was hyperhidrosis affected by posture. The pathophysiology involved and the management of these patients is discussed.
\end{abstract}

Keywords: hyperhidrosis; post-traumatic syringomyelia; autonomic dysreflexia.

\section{Introduction}

Profuse sweating or hyperhidrosis $(\mathrm{HH})$ is one of the many complications that can occur after spinal cord injury. It may occur as an isolated symptom without any associated etiology, as part of the autonomic hyperreflexia syndrome, or as a manifestation of post-traumatic syringomyelia (PTS). ${ }^{1}$ Two cases are presented in which the first and predominant symptom of PTS was postural induced $\mathrm{HH}$.

\section{Case reports}

\section{Case 1}

This patient was a 17 year old male who sustained a C5 vertebral burst fracture while playing ice hockey on $12 / 7 / 83$. At his initial presentation he was a C5 complete, Frankel's A quadriplegic. He underwent a posterior cervical fusion with iliac bone graft, followed by halo immobilization. Over time he gained one motor level to become a C6 level quadriplegic. The patient's subsequent course was complicated by

\footnotetext{
*This paper was presented at the 39th Annual Conference of the American Paraplegia Society, Las Vegas, Nevada, September 7-9, 1993

${ }^{\dagger}$ Correspondence: Department of Neurosurgery, Erie County Medical Center, 462 Grider Street, Buffalo, New York 14215, USA.
}

autonomic hyperreflexia due to bladdersphincter dyssynergia, which was ultimately successfully treated with a suprapubic catheter.

In 1987 he developed severe $\mathrm{HH}$ which always occurred when sitting upright and was relieved in the recumbent position. The sweating was more pronounced in the face and upper extremities than in the trunk or the lower extremities. The $\mathrm{HH}$ was accompanied by a right-sided Horner's syndrome but no other manifestations of autonomic hyperreflexia. Gastrointestinal and genitourinary investigations were negative and there was no skin breakdown.

An MRI on 11/30/87 revealed a posttraumatic syrinx extending from $\mathrm{C} 4-\mathrm{C} 5$ to T7-T8. (Figs 1a, b) Subsequently, he underwent C5-C6 and T5-T6 laminectomies with placement of a syringo-subarachnoid shunt. Intraoperative ultrasound at the cervical level showed collapse of the cyst. Postoperatively, there was significant improvement in the patient's symptom and the right Horner's syndrome disappeared. No MRI was performed.

The patient did well until 1989 when he again developed $\mathrm{HH}$ in the upright position associated this time with a mild increase in spasticity and recurrence of the right Horner's syndrome. An MRI was repeated on 3/7/89 and showed a persistent large syrinx from C5 to T6. The syrinx appeared to be the same size as was demonstrated in his preoperative MRI, implying malfunction of the drainage tube. On $7 / 6 / 89$ the T5-T6 laminectomy was reopened and the syringo-subarachnoid shunt was revised. 


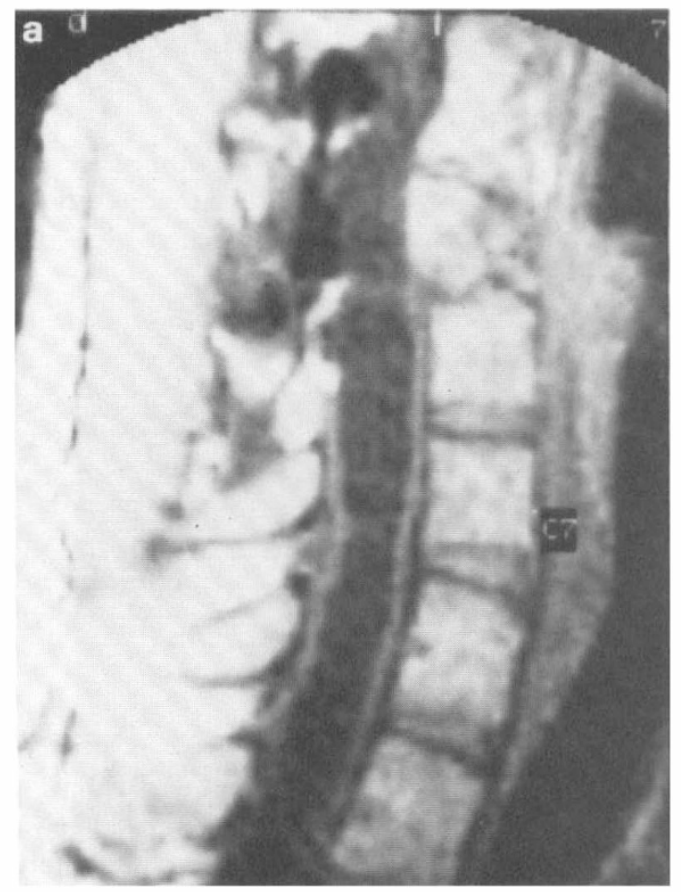

Figure 1 Case 1. MRI scan of (a) cervical and (b) upper thoracic spine 11/30/87 demonstrating an extensive syrinx cavity from $\mathrm{C} 4$ to $\mathrm{T} 8$.

Again, postoperatively there was significant improvement in his positional $\mathrm{HH}$, although not complete resolution. A repeat MRI showed significant decrease in the size of the syrinx and subsequent MRI studies have shown a stable syrinx size with minimal changes. (Fig 2)

\section{Case 2}

A 27 year old female was involved in a motor vehicle accident on 6/26/78 resulting in a compression fracture of the $\mathrm{C} 6$ vertebral body. On neurological examination she was a $\mathrm{C} 6$ complete Frankel's A quadriplegic. She was treated initially with skeletal traction for 6 weeks and then had an external orthosis. In the course of her intensive rehabilitation she developed severe cystitis, and a sacral decubitus sore requiring a rotation flap operation. Spasticity was initially treated with dantrium.

Her normal sweat pattern involved both upper extremities, face and the neck anteriorly down to the clavicle and posteriorly to the lower portion of the scapulae. She experienced the typical sympathetic sweating (autonomic dysreflexia) with the occurrence of bowel or bladder problems. She also developed mild ab-

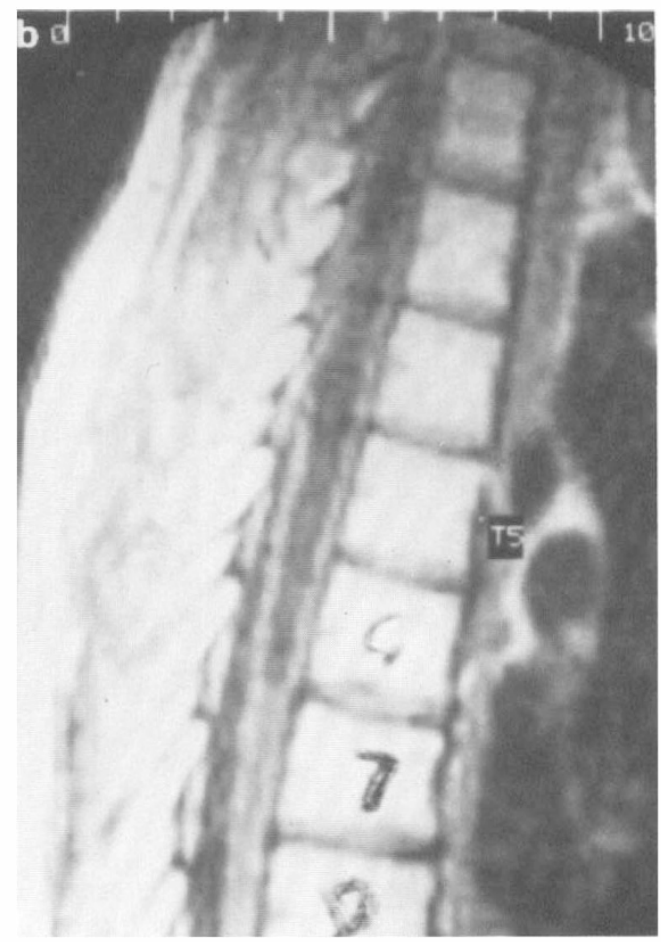

dominal spasms as well as spasms of the lower extremities which, however, were not interfering with her activities of daily living.

In September 1991, she developed HH coming on either immediately or within one-half hour after being in the upright position. It would also happen when she was lying on her right side. The $\mathrm{HH}$ occurred in the area of her normal sweating: namely her head and shoulders and would be so profuse that her clothes and towels were soaked. Again, no other manifestations of autonomic dysreflexia were present. Changing to the supine position or lying down would instantly stop the HH. In addition, she noted some tingling in both hands, more marked in the fingertips and a vibratory sensitivity of her bones, especially around the waist. There may have been a very mild increase in her spasticity. However, sensation and the strength in the upper extremities remained unchanged.

An MRI in February of 1992 demonstrated a post-traumatic syrinx extending from $\mathrm{C} 2 \cdot$ to about the midthoracic region $\mathrm{T} 8$. In the area of her vertebral injury the spinal canal was somewhat encroached upon by the deformed bone (Fig 3). 


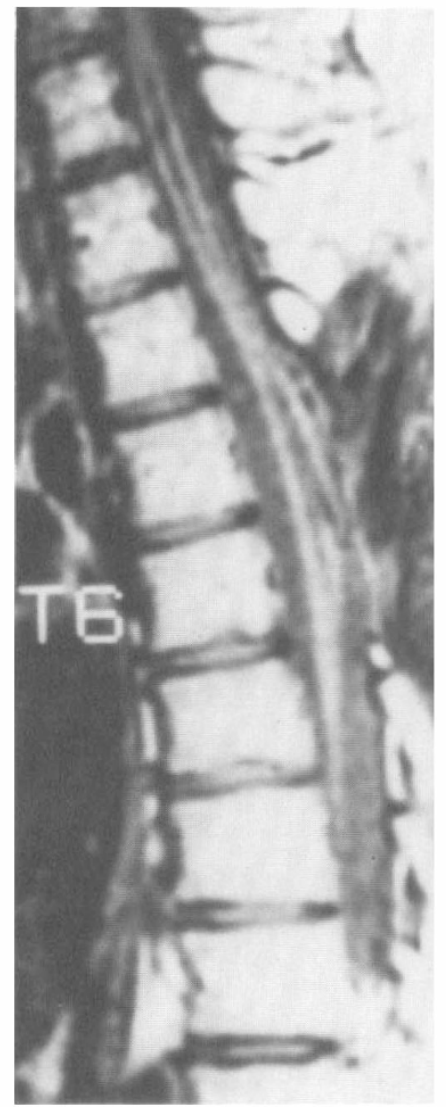

Figure 2 Case 1. MRI of thoracic spine in June 1990, demonstrating considerable collapse of the syrinx after successful shunt revision.

A syringo-subarachnoid shunt at the upper thoracic spinal level was carried out 6/29/92. She had immediate short term relief of her $\mathrm{HH}$ for about 1 month, followed by recurrence of her symptom.

A repeat MRI in September of 1992 again showed the presence of the syrinx but the cavity was slightly narrower.

In November 1992 she reported complete relief of her $\mathrm{HH}$ for about 3 months following a bout of bronchitis. This occurrence was attributed to a clearing of a possible obstructed shunt tube from debris by the increased intraspinal pressure during coughing. Subsequently, her $\mathrm{HH}$ returned and an MRI in January of 1993 showed the syrinx to be enlarged, extending from $\mathrm{C} 2$ to $\mathrm{T} 10$.

In March 1993 the previous syringosubarachnoid shunt was converted to a syringoperitoneal shunt. At the time of surgery the distal portion of the shunt catheter in the

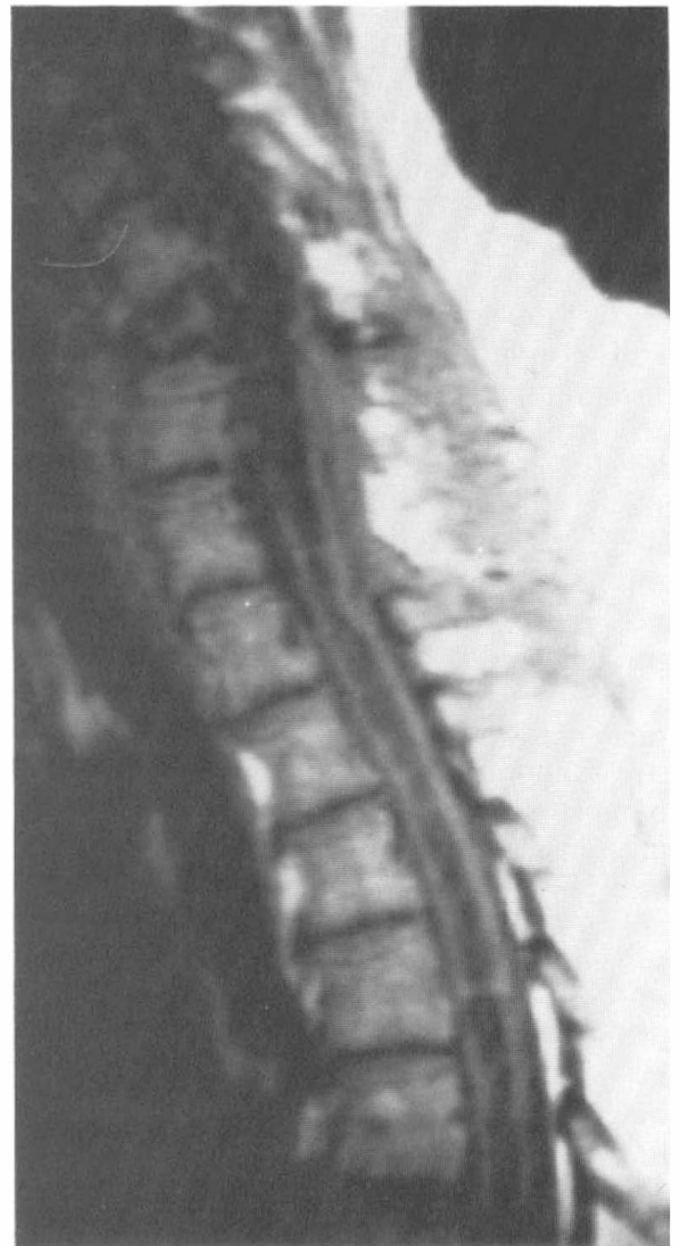

Figure 3 Case 2. Preoperative MRI of cervical and upper thoracic spine confirms extensive syrinx from $\mathrm{C} 2$ to $\mathrm{T} 8$ and encroachment of spinal canal by bony deformity at C6.

subarachnoid space was found to be tethered and enveloped by scar tissue. After this procedure there was considerable improvement in her symptoms. Presently there is mild sweating only after being upright for a couple of hours compared to the immediate onset prior to surgery and no $\mathrm{HH}$ when she is in the right lateral position.

\section{Discussion}

PTS occurs in a small percentage of spinal cord injured patients. Herz et $a l^{2}$ reported that among 850 individuals referred to a rehabilitation center, five patients devel- 
oped new symptoms due to PTS, an incidence of $0.6 \%$. Oakley et $\mathrm{al}^{3}$ in 1981 reviewed the literature and recovered 93 cases of PTS. It occurred in about $1.8 \%$ of paraplegics and less than $0.5 \%$ of quadriplegics. A similar incidence was quoted by Barnett et al. ${ }^{4}$ In Rossier et al's ${ }^{5}$ series of 951 patients with spinal cord injury, 30 $(3.9 \%)$ developed cervical syringomyelia. This condition was found in $22(4.5 \%)$ post-traumatic quadriplegic and $8(1.7 \%)$ post-traumatic paraplegic patients. The incidence of cervical syringomyelia was about $8 \%$ in patients with complete quadriplegia. However, greater awareness of this entity and the increased use of MRI will result in an earlier diagnosis and in diagnosing a greater number of patients.

The interval between trauma and the presentation of a syrinx varies widely, from several months to many years. ${ }^{2,6,7}$ In the patients reported here the interval of spinal injury to the onset of symptoms was 4 years and 13 years respectively. The interval for appearance of the syrinx is, somewhat shorter in those with complete compared to those with partial spinal cord lesions. Most of the responsible injuries for PTS occurred in the lower cervical or high thoracic area but may also be associated with low thoracic or even upper lumbar lesions.

The syrinx cavity can either ascend or descend within the cord from the site of damage and not infrequently is found above and below the spinal cord lesion. ${ }^{8}$ However, in almost all cases the upper thoracic cord is involved.

Pain, various sensory changes, progressive motor weakness, and increase in spasticity are the most frequent symptoms of PTS. ${ }^{4,7}$ Excessive sweating or $\mathrm{HH}$ is not an early feature of PTS. Piatt ${ }^{7}$ does not mention $\mathrm{HH}$ at all in his description of PTS. Batzdorf $^{6}$ reported on several patients whose symptoms of syringomyelia were triggered by recent trauma and mentions abnormalities of sweating occuring in the upper limbs and upper face or the occasional lack of sweating on one side or the other in syringobulbia. If Horner's syndrome was present it occurred usually unilaterally. He referred to one patient with persistent and distressing $\mathrm{HH}$. Rossier's series of 30 patients with PTS included four patients with symptoms of increased sweating and only in one patient was $\mathrm{HH}$ the initial symptom. ${ }^{5}$ Anderson et $a l^{1}$ in their report on $\mathrm{HH}$ emphasized that current $\mathrm{HH}$ problems were reported by 56 patients among 192 spinal cord injured patients. Six of the patients reported the onset of very annoying $\mathrm{HH}$ 2-8 years after their spinal cord lesion. Barnett et $\mathrm{al}^{4}$ observed segmental $\mathrm{HH}$ in eight of their 17 patients with PTS. Sweating was severe enough that patients commented on it spontaneously and three patients required frequent daily changes of clothing. However, in only one patient was $\mathrm{HH}$ the initial symptom of PTS whereas in the other patients increased sweating appeared to be a presentation of autonomic dysreflexia. Only Stanworth ${ }^{8}$ reports on the significance of $\mathrm{HH}$ in eight patients with PTS. In two of his patients $\mathrm{HH}$ was the first presenting symptom. The excessive sweating occurred below the lesion in five and above the lesion in three patients. Unilateral sweating occurring in three was provoked by lying in the lateral decubitus position. Other reports rarely refer to $\mathrm{HH}$ as a presenting symptom.

If $\mathrm{HH}$ is present it occurs in such excessive quantities that, for example, pools of sweat accumulate in the bed, or sweat can be wrung out of clothes and towels. It may occur above or below the level of transection, unilaterally, and may involve dermatomes whose sensation is later altered by the syrinx. ${ }^{8}$ In our two patients $\mathrm{HH}$ occurred primarily above the lesion and bilaterally.

Sweat glands derive their innervation from the sympathetic nervous system. The hypothalamus and other suprasegmental structures contribute to the regulation of a variety of visceral reflex activities through the descending fiber system. Within the spinal cord the supranuclear sudomotor fibers pass through the posterior aspect of the anterolateral tract very close to the lateral pyramidal tract and the border zone of the intermediolateral horns where the segmental synapse with the ganglion cells of the gray matter takes place. The intermediolateral nucleus consists of several adjacent cell columns, the most apical cell group constituting the lateral horn (Fig 4). It 


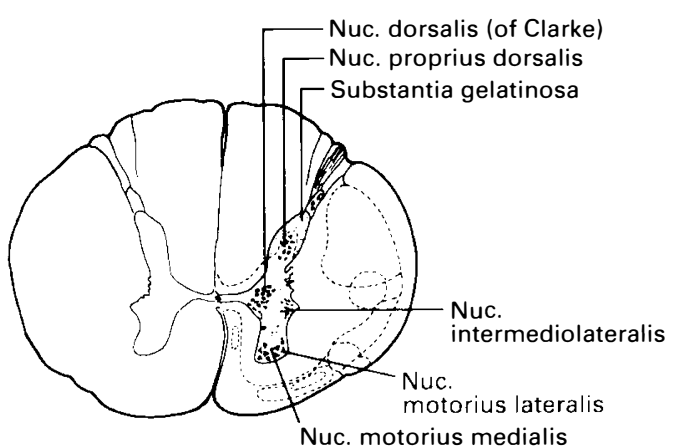

Figure 4 Cross-section of spinal cord at upper thoracic (T5) level.

contains preganglionic sympathetic neurons and is limited to thoracic and lumbar levels T1-L2. ${ }^{9,10}$ Laminae 7 and 8, which include the dorsal nucleus of Clarke, extend throughout the zona intermedia and ventral portions of the gray matter. The intermedial cell column is associated with visceral motor reflexes. ${ }^{11,12}$

The peripheral processes of these cells give rise to preganglionic sympathetic fibers which emerge from the spinal cord via the ventral roots (Fig 5). Peripherally these fibers leave the mixed spinal nerve as the white rami communicantes, enter the sympathetic trunk and terminate upon cells in the paravertebral or prevertebral ganglia.

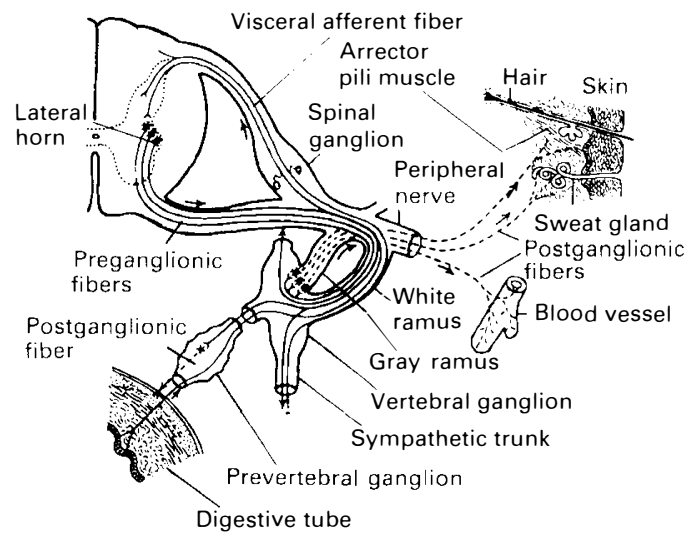

Figure 5 Diagram of sympathetic reflex arcs (reproduced with permission of the author from Carpenter MD \& Sutin J (1983) Human Neuroanatomy, figure $8-2$, p. 212 , Williams \& Wilkins, Baltimore).
Those terminating in the paravertebral ganglia either end there or terminate in ganglia above or below the level of their entrance. While the white rami communicantes are limited to the thoracic and upper lumbar nerves, each spinal nerve receives a gray ramus communicans from the sympathetic trunk. The gray ramus consists of unmyelinated, sympathetic postganglionic fibers which supply the dilator muscles of blood vessels, sweat glands, the hairs of the head and face, and the lacrimal and salivary glands. ${ }^{9}$

The distribution of the postganglionic sympathetic fibers compares closely to that of the sensory dermatomes. ${ }^{13,14}$ However, since sympathetic innervation does not follow a clear segmental distribution, normal sweating may be preserved at a higher or lower level than skin sensation. ${ }^{10}$

Although the thoracolumbar outflow arises from a cell column in a restricted region of the spinal cord, it represents the total sympathetic output for the entire body. Lesions of the descending central tracts can result in complete loss or altered control of visceral activities at lower segmental levels. Complete interruption of the sudomotor pathways abolishes the thermoregulatory function of the sweat glands resulting in anhidrosis. ${ }^{13}$ In the cervical cord there is a somatotopic arrangement within the supranuclear sudomotor tract which is similar to other tracts.

Most viscera receive a double autonomic innervation with the notable exception of the glands of the skin and peripheral blood vessels which receive only a sympathetic innervation. The sweat glands of the head and neck are supplied by the intermediolateral nucleus in cord segments T1-T3.9,10

The neurohumoral transmitter of all preganglionic autonomic fibers, of all postganglionic parasympathetic fibers, and of postganglionic sympathetic nerve fibers innervating sweat glands is acetylcholine. Overactivity of both the adrenergic and cholinergic fibers results in autonomic imbalance.

In patients with spinal cord injury the afferent impulses from below the level of the lesion enter the spinal cord through the dorsal nerve root and ascend along the 
spinothalamic tracts or the dorsal columns. At segmental levels up to the level of the lesion the impulses may cause a spinal mass reflex, referred to as autonomic hyperreflexia or autonomic dysreflexia. Autonomic hyperreflexia has been suggested to be the result of sprouting of ascending fibers forming abnormal synapses in the intermediolateral gray columns and resulting in mass discharge of sympathetic neurons from peripheral stimuli. ${ }^{1}$ It is usually the result of metabolic or mechanical irritation below the level of the lesion such as bladder or bowel distention, pressure sores, or infection. This can result in hypertension, alterations in gastrointestinal motility, bradycardia, and vasodilatation proximal to the level of the lesion. Sweating is one of the manifestations of autonomic hyperreflexia. For unknown reasons there seems to be a head and neck predominance of sweating during autonomic discharge. The lowest level most often mentioned in which autonomic hyperreflexia has been seen is T6 but cases even at levels down to T11 have been reported. ${ }^{1,10}$

A change of posture especially in cervical lesions may evoke reflex sweating. This may occur when a patient is in the prone position or lying on the side. It is also observed when a quadriplegic patient is raised from a horizontal to upright position, in particular when standing in the parallel bars.

Frequently, only part of the sympathetically innervated functions are activated. Thus, in the autonomic crisis, drenching sweat may occur without vasospasm. This can be explained by the presence of distinct fiber tracts subserving sudomotor and vasomotor functions. ${ }^{10}$

Anterior horn lesions are not infrequently combined with vasomotor disturbances of sweating. The classical representation of central damage or destruction of the spinal cord is syringomyelia.

$\mathrm{HH}$ develops above the level of complete cord lesions. The exaggerated activity of sweat glands is particularly conspicuous in the segments nearest the lesion and reveals itself by early onset of increased sweating in a bandlike form. The sympathetic mechanism can be considered to be disinhibited but locally intact comparable to the intact muscle stretch reflex mechanism which is hyperactive after section of the corticospinal tracts. ${ }^{15}$

The $\mathrm{HH}$ occurred in our two patients primarily when in an upright position and in the second patient also when she was on her right side and was promptly abolished by change or in the recumbent position. This change of position is indicative of pressure changes within the syrinx especially in the upright or other position when the hydrostatic pressure in the 'syrinx tube' may result in irritation or pressure on the adjacent intermediolateral tract or nucleus. Septation in the syrinx and adjacent scarring of the spinal cord can contribute to this phenomenon rather than the postural influence on epidural veins. Percutaneous puncture of the syrinx has been carried out for the diagnosis and treatment. ${ }^{16-18}$ Direct measurements of the syrinx pressure have been found to be elevated especially in cases of septation which may represent loculation or an evolving picture in the progression of the syrinx and is frequently found to be communicating. ${ }^{17,18}$ Increase in ventricular pressure has been observed to increase the pressure within the syrinx or cyst. The transmission of the thoracic pressure to the spinal subarachnoid space with compression of the syrinx is thought to be the principal force that enlarges the syrinx. 19

Different methods of treatment for $\mathrm{HH}$ have been used with variable results. Among them is the use of acupuncture and a short course of epidural morphine injection. Methantheline bromide diminished excessive sweating but never abolished it. ${ }^{1.15}$ In one study topical scopolamine successfully controlled reflex sweating without anticholinergic side effects. ${ }^{20}$

Direct drainage of the syrinx or various shunting procedures, preferably a syringoperitoneal shunt are the most successful surgical procedures in the treatment of PTS. The use of intraoperative ultrasound for the exact placement of the shunt catheter has been recommended by Wilberger et al. ${ }^{21}$ Drainage of the syrinx via a shunting procedure either abolished or markedly improved the $\mathrm{HH}$ in our two patients. Malfunction of the drainage system resulted in re-expansion of the syrinx and recurrent HH. Subsequent improvement after 
revision of the syrinx shunt supports the anatomical and pathophysiological events and explains the presenting symptom of $\mathrm{HH}$.

$\mathrm{HH}$ can be an early and significant symptom in the development of PTS. Spinal cord injured patients frequently have a precarious independence and, therefore, are easily threatened by additional loss of function. Early detection of the development of PTS is very important in the diagnosis and prompt surgical treatment.

\section{Summary}

PTS usually occurs in those with a low cervical spinal cord injury especially with a complete neurological lesion. Symptoms oc- cur several years after the injury. The syrinx extends either above and/or below the level of injury and almost always involves the upper thoracic spinal cord. $\mathrm{HH}$ is not a frequent symptom and is especially rare as a presenting symptom of PTS. If $\mathrm{HH}$ is present it occurs in excessive quantities and is affected by posture. The symptoms are promptly relieved by surgical drainage of the syrinx.

\section{Acknowledgements}

We wish to thank Paul $\mathrm{H}$ Dressel from the Department of Neurosurgery for the preparation of the illustrations for this article, and Judy Berst from the Department of Rehabilitation Medicine for preparation of the manuscript.

\section{References}

1 Andersen LS, Biering-Sørensen F, Muller PG et al (1992) The prevalence of hyperhidrosis in patients with spinal cord injuries and an evaluation of the effect of dextropropoxyphene hydrochloride in therapy. Paraplegia 30: 184-191.

2 Herz DA, Gregerson M, Pearl L (1986) Rehabilitative neurosurgery. Neurosurgery 18: 311-315.

3 Oakley JC, Ojemann GA, Alvord Jr EC (1981) Post-traumatic syringomyelia. Case report. J Neurosurg 55: 276-281.

4 Barnett HJM, Foster JB, Hudgson P (1973) Syringomyelia. W B Saunders Co Ltd, London.

5 Rossier AB, Foo D, Shillito J et al (1985) Post-traumatic cervical syringomyelia. Brain 108: 439-461.

6 Batzdorf U (editor) (1991) Syringomyelia: Current Concepts in Diagnosis and Treatment. Williams \& Wilkins, Baltimore: 91-115.

7 Piatt Jr JH (1985) Post-traumatic syringomyelia. In Wilkins RH \& Rengachary SS, editors. Neurosurgery. Vol II. McGraw-Hill Book Co, New York: 1761-1763.

8 Stanworth PA (1982) The significance of hyperhidrosis in patients with post-traumatic syringomyelia. Paraplegia 20: 282-287.

9 Carpenter MB, Sutin J (1983) Human Neuroanatomy. Williams \& Wilkins, Baltimore.

10 Fast A (1977) Reflex sweating in patients with spinal cord injury: A review: Arch Phys Med Rehabil 58: 435-437.

11 Burt AM (1993) Neuroanatomy. W B Saunders Co Ltd, London.

12 Haines DE (1983) Neuroanatomy. An Atlas of Structures, Sections and Systems. Urban \& Schwarzenberg, Baltimore-Munich.

13 Guttmann L (1976) Spinal Cord Injuries. Comprehensive Management and Research. Blackwell Scientific Publications, London.

14 Huckaba CE, Frewin DB, Downey JA et al (1976) Sweating responses of normal, paraplegic and anhidrotic subjects. Arch Phys Med Rehabil 57: 268-274.

15 Kneisley LW (1977) Hyperhidrosis in paraplegia. Arch Neurol 34: 536-539.

16 Dietemann JL, Babin E, Wackenheim A et al (1982) Percutaneous puncture of spinal cyst in the diagnosis and therapy of syringomyelia and cystic tumors. Neuroradiology 24: 59-63.

17 Ellertsson A, Greitz T (1970) The distending force in the production of communicating syringomyelia. Lancet 1: 1234.

18 Lederhaus SL, Pritz MB, Pribram HFW (1988) Septation in syringomyelia and its possible. clinical significance. Neurosurgery 22: 1064-1067.

19 Hall P, Turner M, Aichinger S et al (1980) Experimental syringomyelia. The relationship between intraventricular and intrasyrinx pressures. J Neurosurg 52: 812-817.

20 Staas Jr WE, Nemunaitis G (1989) Management of reflex sweating.in spinal cord injured patients. Arch Phys Med Rehabil 70: 544-546.

21 Wilberger Jr JE, Maroon JC, Prostko ER et al (1987) Magnetic resonance imaging and intra-operative neurosonography in syringomyelia. Neurosurgery 20: 599-605. 